\title{
IMPORTANCIA DE LA BIOÉTICA EN LA FORMACIÓN DEL ABOGADO. EL CASO COLOMBIANO
}

Julieta Melo Correa*

Recibido: 06.09.2014

Aprobado: 04.10.2014

\section{RESUMEN}

Estudio sobre la importancia que debe tener la Bioética en la formación de los abogados en Colombia, específicamente en Cali; el objetivo principal es comprender la importancia de su formación en temas bioéticos, con la finalidad de establecer una propuesta de formación académica concebida para abogados. Se analizaron categorías como: dilemas bioéticos con la aplicación de los avances científicos, multidisciplinariedad bioética y derecho, aportes de la bioética en el desarrollo humano del abogado, razones por las cuales debe formarse el abogado en bioética. En los resultados se encontró un predominio del enfoque, cualitativo desde lo analítico, y el carácter de este lo otorgó el escaso número de investigaciones en torno al tema, evidenciándose una crónica carencia formativa de los abogados en asuntos bioéticos como consecuencia de la precaria y casi nula oferta académica, dejando al descubierto como conclusión, la desactualización e incapacidad del abogado para afrontar el profundo desafío de la ciencias, imponiéndose la necesidad de insistir en la validez de su formación y, por tal razón, debe trascender a la comunidad jurídica a través de su institucionalización.

\section{PALABRAS CLAVE}

Bioética - Derecho - Bioderecho - Interdisciplinariedad - Áreas de impacto en el estudio de la bioética - Corrientes bioéticas - Dilemas fundamentales - Interrogantes jurídicos - Enseñanza de la Bioética.

\section{ABSTRACT}

The importance it should have bioethics in the training of lawyers in Colombia in Cali was analyzed specifically; the main objective is to understand the importance of training in bioethical issues for the purpose of establishing a proposal for academic training designed for lawyers. Bioethical dilemmas with the application of scientific advances, multidisciplinary bioethics and law, bioethics contributions in human development attorney, why the attorney should be trained in bioethics: as categories were analyzed. In the results a predominance of the approach, qualitative found from the analytic and the character of this so it granted the limited amount of research on the subject, demonstrating a chronic lack of training of lawyers in bioethical issues as a result of the precarious and almost no academic offerings, revealing conclusion, the downgrade and inability to cope with the profound lawyer challenged the sciences, imposing the need to insist on the validity of their training and for that reason should transcend the legal community through its institutionalization.

\section{KEYWORDS}

Bioethics - Law - Biolaw - Interdisciplinary - Impact areas in the study of bioethics - Currents in bioethics - Fundamental dilemmas - Legal questions - Teaching of Bioethics.

Abogada colombiana. Egresada de la Universidad Cooperativa de Cali. Especialista en Derecho de Familia en la Universidad Pontificia Bolivariana. Especialista en Conciliación y Arbitraje en la Pontificia Universidad Javeriana. Estudios en Investigación Científica.Especialización y Maestría en Bioética en la Universidad Libre Internacional de las Américas de Chile. Resumen de la tesis con la que optara el grado de Magister en Bioética en la Universidad Libre Internacional de las Américas en el año 2013. 


\section{INTRODUCCIÓN}

La investigación se refiere a la "importancia que debe tener la bioética en la formación del abogado en Colombia", específicamente en la ciudad de Santiago de Cali. El titulo sugiere la formación específicamente del abogado en temas bioéticos, basados en los resultados arrojados durante la investigación, que indican no solo la falta de conocimiento del abogado en dichos temas, sino su desactualización y desinformación en avances tecno-científicos y la tendencia del profesional a considerar que la bioética es propia de la medicina, desconociéndose que desde el surgimiento de la bioética (1970) su enseñanza ha sido un tema central de debate y de profundo interés, lo que obliga a voltear la mirada hacia los abogados y sus procesos de formación pedagógica y didáctica, pues no basta con legislar, sino con alcanzar un determinado nivel de formación que involucre asuntos bioéticos, dado que los dilemas que se presentan frente a los avances científicos, superan la normatividad jurídica, y el abogado no puede sustraerse a esta realidad en asuntos tales como: aborto, eutanasia, clonación, maternidad subrogada, adopción de embriones, eugenesia, esterilización del subnormal, entre muchos otros, donde su tratamiento y búsqueda de respuestas frente a las ilicitudes necesitan de un tratamiento jurídico, coherentes con un modelo bioético.

La característica principal que ha identificado esta investigación es la precaria formación de los abogados en temas bioéticos, debido a la casi nula y escasa oferta académica, generada por razones como: tendencia al relativismo aferrados a un marco jurídico positivista con significaciones clásicas duras de morir, reticencia de los abogados para abordar temas científicos, falta de estímulo en el conocimiento de la bioética. La investigación tiene como principal propósito de estudio que se comprenda la importancia de la formación del abogado en temas bioéticos; su desarrollo se fundamenta en un interrogante: ¿Cuáles son las razones que sustentan la importancia que debe tener la bioética, en la formación del abogado en Colombia?

La investigación del problema formulado se justifica por razones:

- Teóricas: La investigación apunta a desarrollar conocimiento con aplicación de saberes en beneficio de la sociedad, coadyuvar a la retroalimentación y fortalecimiento en la formación integral del ser humano.

- Profesional: Los dilemas bioéticos abordan problemas jurídicos, de los cuales el abogado debe tener conocimiento y formación con capacidad de poder articular su profesión a las transformaciones científicas.

- Práctica: De acuerdo con los objetivos de la investigación, sus resultados permiten explicar las razones concretas por las cuales se debe formar el abogado en temas bioéticos, y su importancia, impulsando de esta manera la comprensión de las razones que lo fundamentan.

\section{Metodología}

La investigación se lleva a cabo a través del enfoque cualitativo mediante el análisis. Las fuentes primarias son las entrevistas transcritas. El estudio se realizó en la ciudad de Santiago de Cali y la población muestra fue tomada de las Facultades de Derecho. Para dar respuesta al objetivo general, se desarrollaron como categorías independientes los siguientes temas: Análisis de algunos de los dilemas que se presentan con la aplicación de los avances científicos y que requieren de la formación del abogado. La multidisciplinariedad bioética y derecho. Aportes de la bioética en el desarrollo humano del abogado. Razones por las cuales debe formarse el abogado en temas bioéticos. Proponer la enseñanza de la bioética en las Facultades de Derecho en Cali.

\section{Problema}

Se pretende a través de esta investigación, comprender las razones que fundamentan el papel de la bioética en la formación del abogado mediante la propuesta titulada: Importancia que debe tener la Bioética en la formación del abogado en Colombia, específicamente en Santiago de Cali, por cuanto en esta ciudad, en el ámbito de las Facultades de Derecho ${ }^{1}$, se evidencia una notable precariedad en la oferta académica en temas bioéticos, prevaleciendo la desconexión de las realidades éticas y científicas.

\footnotetext{
En Santiago de Cali - Colombia, existen seis Facultades de Derecho: Universidad San Buenaventura, Universidad Cooperativa de Colombia, ICESI, Universidad Santiago de Cali, Universidad Libre, Universidad Javeriana.
} 
Ante tan importante déficit es comprensible que exista dificultad para la asimilación y comprensión de algunos conceptos básicos que permitan el ejercicio de la lógica argumental con fundamentos éticos serios. ${ }^{2}$ Tal situación de precariedad en su oferta es originada por: multiplicidad de opiniones acerca de valores ${ }^{3}$, generalización de la idea de que la bioética es exclusiva de la salud, pero específicamente la razón más marcada es la tendencia al relativismo, aferrados a un marco jurídico ajeno al debate científico, con conceptos clásicos duros de morir ${ }^{4}$, reticencia en asuntos científicos, desinterés en el conocimiento y aprendizaje de la bioética; lo anterior indica que el abogado no está preparado para enfrentar situaciones que se presentan frente al avance de la biotecnología.

Dicho ciclo de transformación científica, obliga al abogado a reflexionar sobre los valores y la formación académica en bioéti$\mathrm{ca}^{5}$ para poder asumir los retos jurídicos que plantea la biotecnología, pues no basta con legislar con normas que hoy se presentan como omnipotentes y obsoletas, al lado de la memorización, generándose con ello la incapacidad del abogado de visualizar y mucho menos de afrontar el profundo desafío de los avances de la ciencia sobre asuntos técnicamente comple- jos; por tales razones, se hace necesario que las Facultades de Derecho en Cali, deban buscar enseñar la bioética, constituyéndose en una nueva sabiduría según Potter ${ }^{6}$,de tal manera que ello pueda contribuir a la humanización del derecho.

\section{FormulaCión DEL PROBLEMA}

¿Cuáles son las razones que fundamentan la importancia que debe tener la bioética, en la formación del abogado en Colombia, específicamente en Cali?

\section{Objetivos}

- General. Comprender las razones que fundamentan la importancia que debe tener la bioética, en la formación del abogado en Colombia, específicamente en Cali.

- Específicos. Analizar algunos de los dilemas que se presentan frente a los avances de la ciencia y que requieren la formación bioética del abogado. Analizar la multidisciplinariedad: bioética - derecho y bioderecho. Analizar los aportes de la bioética en el desarrollo humano del abogado. Analizar cuáles son las razones por las cuales debe formarse el Abogado en temas bioéticos. Proponer la enseñanza de la bioética en los diferentes programas de Derecho en Cali.

\section{MARcos de Referencia}

ESTADO DEL ARTE. Se presenta una síntesis de algunas obras, diversos ensayos y artículos relacionados con el tema y que sirven como referente para la solución del problema, de los cuales se resaltan los siguientes: la Facultad de Derecho de la Universidad Cooperativa de Colombia, en Cali, por medio de la investigadora lideró tesis de grado sobre temas bioéticos relacionados en los objetivos específicos y que hacen parte de los dilemas que se presentan frente a los avances de la ciencia, tales como: técnicas de fertilización heteróloga sin consentimiento del cónyuge o compañero permanente (2006); efectos jurídicos de la clonación en Colombia (2007); efectos jurídicos de la maternidad por sustitución en Colombia (2007); creación de un marco legal que consagre la adopción de embriones en Colombia (2008); evolución del concepto de maternidad - paternidad a la luz de las técnicas de fertilización en el derecho de familia en Colombia (2008).

En cuanto a la multidisciplinariedad de la bioética, se desarrollaron los siguientes temas: la juridicidad de la bioética con la integración de los principios del bioderecho y bioderecho de familia (2009); el bioderecho en Colombia (2008); a nivel nacional están los escritos de la

2 ATIENZA, Manuel (1999), “Juridificar la bioética. Una propuesta metodológica”, en Cambrón, A. (Coord.). Entre el nacer y el morir. Granada: Comares, p. 45 y 55.

3 La investigadora, Doctora Julieta Melo, dictó unas conferencias en Bioética y Derecho en la Universidad Autónoma de Cali, pero no se le permitió abordar algunos casos en Bioética por considerar posible adoctrinamiento.

4 ESSER, Josef. Citado por la Doctora NARANJO RAMIREZ, Gloria Patricia (2006). En Libro: Investigación en genética humana y derecho. Universidad Pontificia Bolivariana. Derecho y ciencias políticas. Editorial UPB, p. 19.

5 MOURE GONZALEZ, Eugenio. "La bioética como problema jurídico. Breve análisis de carácter sistémico", Cuadernos de Bioética. No. 28, $4^{\circ} 96$, p. 470.

6 POTTER, Van Rensselaer (1971). Bioethics; bridge to the future. New Jersey. Englewood chiffs, Prantice Hall, p. 205 
doctora Gloria Patricia Naranjo R. de la U.P.B. de Medellín, quien considera la necesidad del abrir el diálogo bioético con otras disciplinas, entre ellas el Derecho, en su libro Investigación en Genética Humana y Derecho (2006). Con respecto la interdisciplinariedad de la bioética, el doctor Favio A Garzón (2010) en Bioética médica y biojurídica, se refiere al interés del derecho frente a la bioética haciendo mención al amparo de las normas jurídicas. El doctor Paulo Arango R. en Consideraciones sobre las implicaciones de la bioética en la Institución Universitaria (1998), se refiere a la bioética en la formación profesional, específicamente en el campo del derecho, al expresar que los avances relacionados con la bioética, superan la normatividad jurídica; en cuanto a los aportes de la bioética en el desarrollo humano del abogado, el doctor Arango (Cuadernos de bioética 1999/1) se refiere a la acción moral sobre el hombre, al manifestar la necesidad de adquirir hábitos buenos llamados "virtudes".

En cuanto a la enseñanza de la bioética, Leonor Rubiano Segura, de la Universidad Tecnológica de Pereira, en ¿Es posible enseñar Bioética? (2010), deja entrever la carencia de la enseñanza en las diferentes Facultades de Derecho de la ciudad de Santiago de Cali. La doctora Elsa Villegas M. (2011), hace un análisis en Tendencia de la formación bioética en los currículos universitarios, argumentando que la inserción de la bioéti- ca en el campo educativo es una realidad urgente.

Internacionalmente, en cuanto a la interdisciplinariedad bioética y derecho, y la formación del abogado en bioética, se encuentran escritos de Susana María Vidal (2010,) en Bioética y desarrollo humano una visión desde América Latina; para María Casado Gonzáles (2002), en Por qué bioética y derecho, manifiesta que es el derecho quien tiene que fijar los límites frente a los avances de la ciencia; el doctor Francisco León Correa (UNESCO 2012), en Antecedentes y situación de la enseñanza de la bioética en Chile, se refiere a la transmisión de los conocimientos en bioética a través de los valores para llegar a cambiar actitudes y comportamientos; en Estado de la enseñanza de la bioética en Argentina, de Miriam M. Sanders (2012), se justifica la enseñanza de la bioética en carreras como el derecho; Igualmente, en $\mathrm{El}$ mundo del derecho frente a las ciencias de la vida: Sociedad del riesgo, derecho y democracia, Cristian Byk (2003) manifiesta el temor a que el derecho sea permisivo frente a las ciencias, $o$ en su defecto que logre limitar o frenar los avances; por su parte, la doctora María D. Vila Coro (2005), considera que el aprendizaje del solo discurso bioético resulta insuficiente frente a la problemática que plantean los avances científicos; Diego Gracia (1989), considera que el bioderecho sin bioética es ciego, y la bioética sin el bioderecho resulta vacía; para la doctora Paula Siverino B. (2012), cuando se refiere a la biojurídica como herramienta para solución de casos complejos, expresa que se está intentando demostrar cómo el quehacer jurídico actual en el Perú, exige de los diferentes operadores del derecho, una formación al menos básica, de nivel informativo en bioética jurídica, ya que temas complejos requieren el manejo de métodos y categorías propias de la disciplina bioética en el marco de los derechos humanos; la doctora Karen Capdet Trinchet (2012), se refiere al abogado y la reflexión bioética como herramienta ética para su buen razonamiento jurídico.

\section{MARCO TeÓRICO}

Para la presente investigación se definieron conceptos establecidos como categorías de los ejes temáticos:

Bioética: Disciplina que se encarga de canalizar, mantener, explicar, proteger, humanizar la adecuada relación entre el hombre, la naturaleza y las técnicas científicas, fomentando el respeto por el medio ambiente y la protección en general del ser humano, considerándolo como fin en sí mismo y no como medio; su objeto es las relaciones entre ciencia, tecnología y sociedad ${ }^{7}$; e interrogar a la ciencia, a fin de establecer los límites, y su finalidad es cuidar y hacer posible la vida como fin en sí mismo.

Dilemas bioéticos. Interrogantes jurídicos: Gloria Patricia

MALDONADO, C.E. (2004). Ciencia y tecnología como política pública y política social. Bogotá: Universidad Externado de Colombia. Observatorio Colombiano de Ciencia y Tecnología.

8 NARANJO RAMIREZ, Gloria Patricia (2006). Investigación en genética humana y derecho. Colombia, ISBN: 958-696-508-2, Vol. Primera edición. Ed. Universidad Pontificia Bolivariana, p. 318. 
Naranjo $(2006)^{8}$, Fabio Alberto Garzón (2010) ${ }^{9}$, Jaime Escobar Triana $(2011)^{10}$ y Miriam Magdalena Sanders $(2012)^{11}$, coinciden en afirmar que el derecho debe ajustarse a la realidad social generada por los avances científicos, con el fin de regular la conducta humana, dado que en la mayoría de los casos no existen parámetros para juzgar asuntos como:

- Técnicas de fertilización asistida heteróloga: Definida según Michelle Sesta (2002) ${ }^{12}$ como la concepción con independencia de la unión física, con aportación de los óvulos del cónyuge y el semen de un tercero. Dilema: ¿La práctica de esta técnica sin el consentimiento del cónyuge se configura como causal de divorcio, o de la disolución de la unión marital de hecho? ¿El donante de semen puede considerarse padre biológico o padre legal? De ser así ¿bajo qué condiciones? ¿Cuáles son los efectos jurídicos con respecto a la filiación cuando no hay consentimiento del cónyuge? ¿Qué hacer cuando no existen normas al respecto?

- Maternidad subrogada: Cuando la mujer que gesta y da a luz aporta su material genético, el cual se fertiliza con el esperma de su marido o de la comitente.

- La maternidad por alquiler de vientre: Mujer que gesta y da a luz, quien no aporta su material genético, pues los aportantes son los comitentes o contratantes. Dilemas que plantean ambas maternidades: ¿Un contrato de maternidad subrogada sería nulo por ilicitud en su objeto teniendo en cuenta el artículo 1523 del Código Civil? ¿Un contrato por alquiler de vientre sería lícito o ilícito por su objeto de contratación? ¿Es posible hablar de legitimidad del hijo con relación a la pareja que lo recibirá una vez nazca? ¿Se presume que el cónyuge de la comitente es el padre del hijo? ¿Puede el cónyuge de la concomitante impugnar la paternidad? ¿Qué sucede si la gestante no desea entregar el bebé aunque exista contrato?

\section{- Fecundación postmortem: ${ }^{13}$} Inseminación de la mujer mediante técnica de fertilización in vitro con semen de su cónyuge o compañero ya fallecido, quien antes de su fallecimiento ya ha crio conservado su semen. Algu- nos dilemas: ¿Puede ser tenido como heredero quien no está en el útero al momento de morir el padre? ¿Podrá realizarse la inseminación sin el consentimiento del cónyuge en vida? ¿Es válida la extracción de la muestra de paciente en estado de coma?

- Clonación: Según Ricardo E. Anton (2002), consiste en que, a partir de una célula somática, se le extrae el núcleo con el ADN del candidato a la clonación y se transfiere al óvulo desnucleado. Algunos dilemas jurídicos: ¿Quien aporta el núcleo, es el padre o madre o hermana? Si la que desea clonarse es mujer sola, esta aportaría su óvulo desnucleado y el núcleo de su célula: ¿Cómo será la filiación del bebé clónico? ¿Es su madre? ¿Es su padre a la vez, o, en su defecto, su hermana ${ }^{14}$ ? Si es pareja lesbiana y una de ellas aporta el óvulo desnucleado y la otra el núcleo de la célula, ¿quién es la verdadera madre? ¿Se trata de dos madres? ¿Se trata de hermanas?

- Investigación con células madres: Según la Doctora Naranjo (2006) $)^{15}$, son células capaces de dividirse, se

9 GARZON, Fabio Alberto. "Bioética médica y biojuridica”. En: Revista Latinoamericana de Bioética [revista en internet] Junio 2010. [Fecha de acceso: 01 Febrero de 2013]. Vol. 10. No. 1 Bogotá. Disponible en: http://www.google.com.co/ webhp?hl=es\&_gc_pw.r:gf.\&fp=b2914588c861e14\&biw=1192\&bih=543.

10 ESCOBAR TRIANA, Jaime Alberto (2011). Propuesta y reflexiones para fundamentar la bioética. Colombia. ISBN: 978-958739-020-9. Vol. 200 Ed. Kimpress Ltda. p. 263.

11 SANDERS, Miriam Magdalena (2012). "Estado de la enseñanza de la bioética". Argentina. En: Revista de bioética latinoamericana [Revista en internet]. Vol. 9. No. 1. p. 79-91. Edición 1. ISBN: 2244-7482. [Acceso: 01 de Febrero de 2013] Disponible en: http://www.saber.ula.ve/bitstream/123456789/34647/1articulo4.pdf.

12 LACADENA, Juan Ramón. GONZALEZ DE CANCINO, Emilsen. ALARCON ROJAS, Fernando y GOYENA COPELLO, Héctor Roberto (2002). Familia, tecnología y derecho. 1ª. Edición. Bogotá: Universidad Externado de Colombia, p. 111-122.

13 RODRIGUEZ RAMOS, Manuel (2002). Familia, tecnología y derecho. Bogotá: Universidad Externado de Colombia. p. 341359

14 ANDORNO Roberto (1998). “La clonación humana y el derecho a la propia identidad genética”. En: La Ley T. 1998-A, Buenos Aires, p. 921.

15 NARANJO RAMIREZ, Gloria Patricia (2006). “Implicaciones jurídicas de la investigación en genética humana”. En: Revista de la Facultad de Derecho y Ciencias Políticas. Medellín, ISBN 0120-3886. p. 431-445. 
extraen de los embriones humanos, ya sea de los embriones supernumerarios de las técnicas de fecundación in vitro. Dilemas: ¿Quién decide hasta cuándo se puede investigar en el ser humano? ¿Es el embrión y el feto cosa o persona? ¿Prima más el criterio de lo científico frente al derecho a la vida y a la dignidad? ¿Por qué la destrucción deliberada de los embriones pudiéndose obtener células madres de la médula ósea de adultos, del cerebro, del bulbo olfativo, retina, hígado, páncreas, así como del cordón umbilical?

- Evaluación de riesgos en los seguros de vida, a partir de tests genéticos: Según la doctora Naranjo (2006), son los análisis de la estructura genética del ser humano, en donde se aprecia las posibles enfermedades o causas de muerte que el individuo pueda llegar a tener. Dilemas: ¿Qué pasa con el derecho a la intimidad de rango constitucional? ¿Qué pasa con los derechos fundamentales de las personas como la no discriminación, o el derecho al trabajo?

- Manipulación genética: La Doctora Naranjo (2006) expresa que esta consiste en cualquier tipo de intervención en el patrimonio genético del individuo (ADN), significando con ello, que el ser humano puede ser manipulado antes de su nacimiento, después, durante su vida y muerte. Algunos dilemas: ¿Puede el científico en aras de la libertad de investigación disponer del código genético de los seres humanos? ¿Estamos frente a una inminente polarización y categorización del ser humano de población genética superior y población genética inferior o inservibles o desechables con las consecuentes discriminaciones, generándose la teoría del etiquetamiento?

- Los testigos de Jehová y su negación a recibir transfusión sanguínea ${ }^{16}$ : Ello, por contrariar sus creencias, lo cual genera un conflicto de valores deberes, derechos, tanto desde la perspectiva de la bioética como jurídico-constitucional, a partir de la disparidad de criterios entre el equipo médico y el paciente que estima necesaria la transfusión sanguínea. Dilemas: ¿Debe prevalecer el respeto de la dignidad humana, aun cuando ello pueda significar poner en peligro la vida de la persona? ¿Es válida la transfusión aun sin el consentimiento del testigo de Jehová?

- La píldora del día después (Levonorgestrel): Se usa durante las siguientes 72 horas después de la relación sexual. Dilemas: ¿Desde qué momento o etapa se exige respeto absoluto por la vida humana? ¿Quién determina la condición de persona: el médico, el jurista, el cientí- fico? ¿Estamos frente a la legalidad de un homicidio?

- Aborto ${ }^{17}:$ La Corte Constitucional colombiana (2006) despenalizó el aborto en tres eventos, pero según Ley del 17 de Diciembre de 2012, se puede abortar por problemas mentales. Interrogantes: ¿Es la Corte Constitucional el órgano legítimo para decidir el valor de la vida y definir el concepto de persona? ¿Los derechos del nasciturus están sometidos al poder del Estado y del derecho positivo? ¿La vida comienza con el nacimiento según el Artículo 90 del Código Civil? ¿Significa esto que el nasciturus no preexiste o no existe la vida? ¿Puede la legislación separar la existencia biológica de la existencia jurídica?

- Eutanasia. Eutanasia Directa: Definida según Francisco León Correa $(1998)^{18}$, como el matar sin dolor y deliberadamente, mediante procedimiento de apariencia médi$\mathrm{ca}$, a personas que se tienen atormentadas por el dolor 0 limitadas por la incapacidad, (activa por acción o pasiva por omisión), o provocar la muerte sin consentimiento a comatosos locos, seniles, vegetales (cacotanasia). Algunos dilemas: ¿Es legítimo plantearse la posibilidad y el deseo de realmente vivir el morir?¿Es mejor dar más vida a los días o más días a la vida? ¿Es la eutanasia un tipo de exterminio eugenésico?

\footnotetext{
Sentencia C-900/11. Expediente D-8523. Ponente Jorge I. Pretel.

Corte Constitucional Sentencia C-355 de 2006. Despenalización del aborto en tres modalidades.

18 LEÓN CORREA, Francisco Javier (1998). "El debate sobre la eutanasia y la medicina actual". Artículo No. 3 Arz. Brevis. En: www.raco.cat/index.php/arsbrevis/article/view/93798/142187.
} 
- La criogenización: Conservación del cadáver congelado a 196 grados de temperatura bajo cero, con el objeto de revertirlo de la muerte en un futuro, cuando la enfermedad por la que falleció, tenga solución. Representa un sin número de interrogantes tales como: ¿Qué pasa con una persona criogénica si es revivida dentro de 100 150 años, cuando aparezca la cura de la enfermedad? ¿Podrá adaptarse a la sociedad después de 150 años?, ¿Qué pasa con los derechos herenciales de estas personas, con su familia? ¿Está viva o muerta realmente? ¿De qué tipo de muerte se está hablando?

- La adopción de embriones: Según María Elsa Petrelli ${ }^{19}$, un número cada vez mayor de embriones, reposan en clínicas de reproducción asistida sin destino definido dado su abandono, a la espera de ser destruidos, o destinados para la investigación, o, en el mejor de los casos, para ser adoptados. Dilemas: ¿Es ilícito utilizar los embriones para experimentos científicos? ¿Cuál es la naturaleza jurídica de la adopción de embriones?

- Interdisciplinariedad: Bioética - Derecho. El tema se desarrolla partiendo de una pregunta: ¿Cómo discutir dilemas bioéticos, si no se poseen criterios tanto bioéticos como jurídicos para resolver- los? La falta de admisión de las anteriores preguntas, da cuenta de un fenómeno: la pretensión y eminente necesidad de la bioética de fusionarse con el derecho según Eugenio More ${ }^{20}$.

Según Aparisi Ángela ${ }^{21}$, cabe preguntarse: ¿Por qué bioética y derecho? Se parte del siguiente fundamento: hay prácticas científicas que pueden resultar potencialmente dañinas al ser humano y por ello se acude al derecho. ¿Por qué el bioderecho? Porque al abordar los temas bioéticos, al surgir diversos conflictos por el uso indiscriminado de las ciencias en el ámbito humano, se abordarían las consecuencias negativas desde la perspectiva de las ciencias jurídicas convirtiéndose el derecho en bioderecho. Dilemas: ¿Cómo discutir en bioética, si no se poseen conocimientos y criterios para resolver las controversias? Lo que significa que el derecho general tiene su propio lenguaje jurídico, Sin embargo, es insuficiente para dar seguridad a los actos que surgen de la aplicación de la ciencia. A partir de lo anterior, se plantea una pregunta: ¿Cuáles son los aportes de la bioética en el desarrollo humano del abogado?

Aportes. El interés por el ser humano en función de su conducta y de su dignidad como persona frente a los demás; al mismo tiempo el estudio de la conducta humana incentivando los valores éticos.
Reflexiones bioéticas como herramienta ética del razonamiento jurídico del abogado. Según Karen Capdet T.22 la bioética coherente con este propósito, ofrece una visión epistemológica que se sostiene con el respeto hacia la dignidad humana como reflexión filosófica, basados en principios de autonomía, justicia, equidad, benevolencia, los cuales se erigen en valores, sobreviniendo como herramienta metodológica, que guía e instruye la ética del razonamiento del abogado. ¿Por qué formarse el abogado en temas bioéticos? El derecho es uno de los campos en el que se debe ejercitar la bioética, por lo tanto, según Severino Bavio y D Agostino, el abogado debe recurrir al abordaje de la bioética para que pueda adquirir un marco conceptual humanizado, de tal forma que este pueda ser capaz de analizar elementos de impacto por la tecnología.

Situación actual de la enseñanza de la bioética en Cali. La mayoría de las universidades de Cali, no contemplan en su plan de estudios, ni en la teoría ni en la práctica, los contenidos de la bioética. Conviene preguntarse si las Facultades de Derecho están graduando profesionales incompetentes desactualizados, bajo un derecho clásico de línea utilitarista, con crónica carencia humanística.

Fundamentación de la enseñanza de la bioética. Como lo manifiestan Viviana Salazar y

19 PETRELLI DE ALIANO, María Elisa (1996). Adopción prenatal. Derecho Civil I y Derecho Civil V. Facultad de Derecho, Santamaría. Buenos Aires: Ponencia Argentina.

20 MORE GONZALEZ, Eugenio (2005). Abogado. Los retos jurídicos que plantea la biotecnología; derecho y salud. Vol. 13. No. 2.

21 APARISI MIRALLES, Ángela. Bioética (2007). "Bioderecho y biojurídica: reflexiones desde la Filosofía del Derecho". En: Anuario de Filosofía del Derecho. Isbn: 0518-0872. Universidad de Navarra: p. 63-83.

22 CAPTDET TRINCHET, Karen (2012). "La reflexión bioética como ética del razonamiento jurídico: aportación del método de construcción del razonamiento”. Derecho general. Universidad de Granma, Cuba: Revista Jurídica, p. 201-203. 
Jenny Bolívar (2010), la enseñanza de la bioética en todos los niveles de educación y campos de formación, se constituye en un imperativo, toda vez que coadyuva no solo a los educadores, sino a los educandos, a dotarlos de herramientas teórico-conceptuales, para la toma de decisiones bien argumentadas, sobre algunos problemas bioéticos, los cuales afectan especialmente al sector vulnerable.

Consideraciones por las cuales debe incluirse la bioética en el plan de estudios de las diferentes Facultades de Derecho. La rapidez de la ciencia y la tecnología obliga a las universidades a replantear los procesos de formación pedagógica y didáctica, teniendo en consideración que el hombre es el único ser en el mundo que requiere de la ética para la convivencia pacífica.

Formación del estudiante. Las Facultades; como instituciones formadoras, deben promover una formación integral holística, con una filosofía basada en la equidad y la justicia, según Miriam Sanders $(2012)^{23}$.

Método de la enseñanza de la bioética. El método puede ser aplicado a la luz de la bioética personalista desde la ética de principios, para poder resolver los dilemas bioéticos a través del ejercicio de la no maleficencia, la beneficencia, la autonomía y la justicia.

\section{ANÁlisis de LoS RESULtAdos}

Se identificaron a la mayoría de docentes con gran sentido de pertenencia a la institución universitaria y alto grado de compromiso con la enseñanza; sin embargo, no dejan de existir aspectos que van en detrimento de la calidad educativa, como el desconocimiento en temas bioéticos, cierta reticencia para acompañar los procesos formativos, y el hecho que pocas universidades propician el debate doctrinario y el estudio de la bioética.

El hecho fundamental que se quiere resaltar es la falta de conocimiento de la bioética por su precaria y casi nula enseñanza académica, tratando de identificar algunas de sus opiniones frente a los dilemas y su aplicación jurídica, lo cual ignoran totalmente los docentes y estudiantes, con marcada tendencia y apego a los conceptos legales de los códigos. Se percibe que no hay una definición de bioética ni un conocimiento claro y profundo sobre el tema, su manejo es muy limitado y lejano, y quienes logran contestar los cuestionarios, hacen parte de los grupos donde la investigadora ha trabajado el conocimiento de la bioética; no se puede hablar de unidad de docentes y profesores en torno a este tema, esta vaguedad en las concepciones obedece más al no conocimiento de la bioética; un alto porcentaje de alumnos y profesores no se atrevieron a responder los cuestionarios referidos a la bioética por falta de conocimiento. En la investigación, con respecto al tema sobre dilemas fundamentales, se dejaron planteados algunos interrogantes, con el fin primordial de despertar inquietudes e interés de los abogados, de tal manera que puedan cuestionar o no su formación de acuerdo a sus respuestas.
Lo anterior permite inferir el vacío formacional en bioética; el abogado no está preparado para enfrentar los desafíos sobre asuntos cruciales de la vida; las tendencias curriculares apuntan hacia el abordaje de temas legales tradicionalistas con habilidades hacia el cobro de honorarios que justifiquen su actuación, existe cierto relativismo y pragmatismo ético, con conceptos de tipo utilitarista.

\section{Conclusiones}

El trabajo de investigación realizado muestra claramente que no hay incursión de la bioética en el campo del derecho; ya sea en el segmento de pregrado como de posgrados; los pocos contenidos que se imparten se realizan sin precisión científica en algunas carreras como la medicina, evidenciándose un vacío tanto de conocimiento bioético como jurídico, donde no existen elementos de juicio para que el abogado pueda abordar los debates en bioética, dado que los procesos de formación de los abogados están dirigidos a resolver asuntos de carácter normativo, de fondos procesales, aferrados a un marco jurídico positivista ajeno a los debates científicos; con crónica carencia humanística, con posturas comunes que se limitan a la tradición académica, la cual trasciende solo a lo estipulado en los códigos, constatándose una creciente demanda de operadores del derecho incapaces de afrontar situaciones dilemáticas vinculadas a los temas bioéticos.

De acuerdo al trabajo de campo realizado, se logró el reconocimiento de los entrevistados 
sobre la importancia que debe tener la bioética en la formación del abogado, siendo este el objetivo general planteado. No se percibe claramente, a corto plazo, la institucionalización y enseñanza de la bioética en un 83\% de las Facultades.
El conocimiento de la bioética debe trascender a la comunidad jurídica a través de su enseñanza. Es necesario incentivar, fortalecer, promocionar la investigación académica y científica, en el campo de la bioética dentro de las Facultades de Derecho.
Impulsar el estudio y formar a los abogados de manera interdisciplinaria, estimulando la teorización, conceptualización y categorización de forma práctica a partir de problemas o dilemas éticos derivados del desarrollo científico. 
